REGULATORY T CELLS

\section{Distilling regulatory T cell inducers}

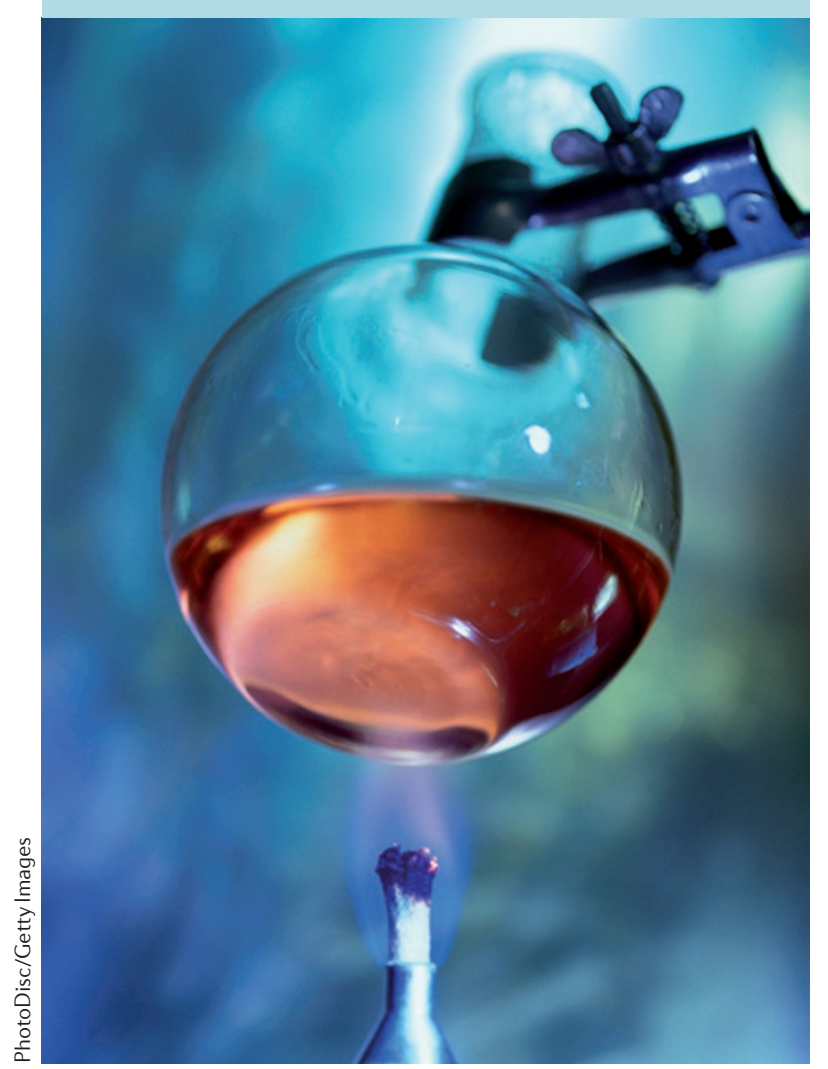

The intestinal microbiota and its fermentation products can influence immunity. Two recent studies report that treatment with microbiota-derived short-chain fatty acids (SCFAs) or with a mixture of 17 bacterial strains from the intestinal microbiota of a healthy individual promotes the expansion and the function of the colonic regulatory $\mathrm{T}\left(\mathrm{T}_{\mathrm{Reg}}\right)$ cell population.

Smith et al. observed that the concentration of SCFAs in the gut is low in the absence of the microbiota. Strikingly, supplementation of germ-free or specific pathogen-free (SPF) mice with SCFAs increased the number of colonic $\mathrm{T}_{\text {Reg }}$ cells and enhanced their expression of the transcription factor forkhead box P3 (Foxp3), interleukin-10 (Il10) and the gut-homing receptor $\mathrm{G}$ protein-coupled receptor 15 (Gpr15). These cells also showed enhanced suppressive activity in vitro. Moreover, supplementation with SCFAs was protective in a mouse model of colitis.

Thus, SCFAs directly affect $\mathrm{T}_{\mathrm{Reg}}$ cells to improve their function, and this activity is dependent on the SCFA-binding receptor GPR43 (encoded by FFAR2), which was shown to be highly expressed by colonic $\mathrm{T}_{\mathrm{Reg}}$ cells. As GPR43 signalling in response to SCFAs resulted in reduced expression of histone deacetylase 6 (HDAC6) and HDAC9, the authors suggest that SCFAs promote $\mathrm{T}_{\text {Reg }}$ cell function through HDAC inhibition.

Atarashi et al. had previously shown that a mixture of Clostridia strains isolated from healthy mice enhances the number and the function of $\mathrm{T}_{\mathrm{Reg}}$ cells in the colon. So, they investigated whether a similar combination of bacterial strains could be isolated from the human intestinal microbiota. The percentage of $\mathrm{T}_{\text {Reg }}$ cells was increased in the gut of germ-free mice that had been orally inoculated with stool samples from a healthy individual, and this effect was greater when the human stool samples had been pretreated with chloroform. Transmission of the chloroform-resistant human microbiota from the primary recipients to secondary mouse recipients through co-housing or reproduction also increased the frequency of colonic $\mathrm{T}_{\mathrm{Reg}}$ cells, even following serial dilution. This indicates that colonic $\mathrm{T}_{\text {Reg }}$ cells can be sustained by a small, stable community of human commensal bacteria.
All 31 bacterial strains that were identified in the caecal content of primary and secondary recipients of the human microbiota belonged to the Clostridia class. Colonization of germfree mice with 17 of these 31 strains, but not with randomly selected combinations of 5 strains, reproducibly enhanced $\mathrm{T}_{\text {Reg }}$ cell numbers in germ-free mice of different genetic backgrounds and in germ-free rats.

The mixture of 17 Clostridia strains that had been identified enhanced not only the proliferation and the gut homing of $\mathrm{T}_{\mathrm{Reg}}$ cells but also their expression of IL-10, inducible T cell co-stimulator (ICOS) and cytotoxic T lymphocyte antigen 4 (CTLA4). Microbiota-derived SCFAs and bacterial antigens were shown to contribute to this improved $\mathrm{T}_{\mathrm{Reg}}$ cell function. Notably, the mixture of 17 Clostridia strains increased the production of active transforming growth factor- $\beta$ which promotes $\mathrm{T}_{\text {Reg }}$ cell proliferation and function - by intestinal epithelial cells through the release of SCFAs.

Finally, the mixture of 17 Clostridia strains decreased the severity of ovalbumin-induced allergic diarrhoea and experimental colitis in germ-free mice that had also been inoculated with the caecal content of SPF mice. This therapeutic effect is relevant, given that at least 5 of these 17 strains seem to be significantly reduced in patients with ulcerative colitis.

These two studies suggest that the therapeutic potential of a combination of probiotics and their derived SCFAs should be evaluated in humans.

Maria Papatriantafyllou

ORIGINAL RESEARCH PAPERS Smith, P. M. et al. The microbial metabolites, short-chain fatty acids, regulate colonic $\mathrm{T}_{\text {reg }}$ cell homeostasis. Science http://dx.doi.org/10.1126/science.1241165 (2013) | Atarashi, K. et al. $T_{\text {reg }}$ induction by a rationally selected mixture of Clostridia strains from the human microbiota. Nature http://dx.doi. org/10.1038/nature12331 (2013) FURTHER READING Kamada, N. et al. Role of the gut microbiota in immunity and inflammatory disease. Nature Rev. Immunol. 13, 321-335 (2013) 